\title{
Synthesis, Characterisation and Photoresponsive Studies of Lignin Functionalised with 2-(5-(4-Dimethylamino-benzylidin)-4-oxo-2-thioxo- thiazolidin-3-yl)-acetic Acid
}

\author{
Ambily Chandran ${ }^{1}$, Sunny Kuriakose ${ }^{1 *}$, Tessymol Mathew ${ }^{2}$ \\ ${ }^{1}$ Research and Post Graduate Department of Chemistry, St. Thomas College, Mahatma Gandhi University, Kerala, India \\ ${ }^{2}$ Research and Post Graduate Department of Chemistry, St. George College, Mahatma Gandhi University, Kerala, India \\ Email: * skresearchgroup@rediffmail.com
}

Received August 24, 2012; revised September 25, 2012; accepted October 5, 2012

\begin{abstract}
A chromophoric system 2-(5-(4-dimethylamino-benzylidin)-4-oxo-2-thioxo-thiazoli din-3-yl)-acetic acid with push-pull electron modulation was synthesised and incorporated onto lignin core (technical lignin, lignin sulphonic acid M.W. $52,400)$ and the photo responsive behaviour was investigated. The product was characterised by UV-visible, fluorescence, FT-IR, and NMR spectroscopic methods. The results of the studies show that the incorporation of the chromophoric system on to the lignin core enhanced the light absorption and light stabilization properties of the chromophoric system. The remarkable stability on irradiation provides a novel photo responsive system with excellent light fastening properties which would find application in coating materials, dyes, paints etc.
\end{abstract}

Keywords: Lignin; Photoresponsive; Environment Friendly; Light Fastening; Coating Material

\section{Introduction}

Lignin is the second most abundant natural polymer, and is obtained as a by product in the pulp and paper industry. Lignin is chemically complex, composed by phenyl propane units connected by various types of linkages, forming a branched structure containing diverse functional groups [1-3]. Lignin is formed by dehydrogenative polymerization of three different types of monomers: pcoumaryl alcohol, coniferyl alcohol, and sinapyl alcohol. Both aliphatic and aromatic hydroxyl group are abundant in it and are potential reactive sites. Because of this multifunctionality, lignin can be considered as a natural polyol [4-9]. In the present study technical lignin is used as the core material. The main source of technical lignin is the black liquor from the pulping process. In the pulping industry, vast amount of lignin are separated from wood and then burned internally in the pulp mill to produce steam and electricity, except for those produced for special chemical purposes.

Photo responsive chromophoric or multi chromophoric systems can be introduced in to the basic structural pattern of lignin by the DCC coupling of free carboxylic groups of chromophoric systems with the end hydroxyl

"Corresponding author. functionalities of lignin. Thus we can develop a photochromic system on to the lignin core and make them able to interact with electromagnetic radiation of selected wave lengths. This could lead to the generation of excellent photoactive systems based on a naturally occurring, environment friendly core material, which find application in paint and dye industries, printing inks, medicine and in medical diagnosis. Functional modification can also change them to molecular nanocapsules and light harvesting antenna systems [10].

\section{Experimental}

\subsection{Materials and Methods: General}

Lignin was purchased from Merck (Germany). Dimethyl amino pyridine (DMAP) and dicyclohexylcarbodiimide (DCC) are commercial samples and used as purchased from E. Merck India Ltd. The solvents used for the study such as dimethyl formamide (DMF) were purified by literature procedure. NMR spectra were recorded on a Bruker $500 \mathrm{MHz}$ NMR instrument. IR spectra were recorded on a Shimadzu FT-IR instrument operating in the range $4000-400 \mathrm{~cm}^{-1}$. UV-visible spectra were recorded on a Shimadzu UV-visible NIR spectrophotometer operating in the range $200-1100 \mathrm{~nm}$. 


\subsection{Synthesis of \\ 2-(5-(4-Dimethylamino-benzylidin)- \\ 4-oxo-2-thioxo-thiazolidin-3-yl)-Acetic Acid}

p-Dimethylaminobenzaldehyde (1 g) and rhodanin- $N$ acetic acid (1.25 g) were dissolved in ethanol. The mixture was stirred thoroughly for a few minutes. The temperature was raised and the mixture was refluxed for 4 hours with magnetic stirring under an inert atmosphere of argon. The product was filtered, purified by recrystallisation from absolute ethanol and further purified by column chromatography using 10:3 chloroform-methanol solvent system and characterised by spectroscopic analysis. The yield is noted as $81 \%$.

\subsection{Synthesis of Lignin Functonalised with 2-(5-(4-Dimethylamino-benzylidin)-4-oxo-2- thioxo-thiazolidin-3-yl)-acetic Acid}

2-(5-(4-dimethylamino-benzylidin)-4-oxo-2-thioxo-thiaz olidin-3-yl)aceticacid(2.25 g), lignin (1 g), DMAP (200 $\mathrm{mg})$, and DCC (1 g) were separately dissolved in DMF and mixed together. The mixture was stirred at room temperature for 2 hours and at $80^{\circ} \mathrm{C}$ for 6 hours. The by-product dicyclohexyl urea (DCU) was removed by warming-cooling-filtration process and the solvents were removed on a vacuum rotory evaporator and dried. It was purified by column chromatography using 10:2 chloroform-methanol solvent system and dried in vacuum. The product was characterised by spectroscopic analysis [11, $12]$.

\section{Results and Discussion}

Lignins are complex, amorphous, phenolic polymers, whose aromatic and aliphatic hydroxyl groups can be easily modified by estrification reaction. Spectroscopic analysis such as UV-visible, FT-IR, and ${ }^{1} \mathrm{HNMR}$ has been done in order to visualize the chemical changes in the structure of lignin.

\subsection{Synthesis and Characterisation of 2-(5-(4-Dimethylamino-benzylidin)-4- oxo-2-thioxo-thiazolidin-3-yl)-acetic Acid}

p-Dimethylaminobenzaldehyde and rhodanin- $N$-acetic acid were condensed together at $80^{\circ} \mathrm{C}$ yielding 2-(5-(4dimethy-amino-benzylidin)-4-oxo-2-thioxo-thiazolidin-3yl)-acetic acid (scheme1).

IR spectrum of 2-(5-(4-dimethylamino-benzylidin)-4oxo-2-thioxo-thiazolidin-3-yl)acetic acid was recorded in the solid state as $\mathrm{KBr}$ discs in the operating frequency range $4000-400 \mathrm{~cm}^{-1}$. The IR spectrum is given in Figure 1. $3440 \mathrm{~cm}^{-1}$ (broad): $v_{\mathrm{O}-\mathrm{H}}(\mathrm{str}), 2920 \mathrm{~cm}^{-1}: v_{\mathrm{C}-\mathrm{H}}$ of $\mathrm{CH}_{2}$, $1716 \mathrm{~cm}^{-1}: v_{\mathrm{C}=\mathrm{O}}(\mathrm{str}), 1573 \mathrm{~cm}^{-1}: v_{\mathrm{C}=\mathrm{C}}(\mathrm{str}), 1379 \mathrm{~cm}^{-1}: v_{\mathrm{C}-\mathrm{N}}$ (str), $1309 \mathrm{~cm}^{-1}: v_{\mathrm{C}=\mathrm{S}}(\mathrm{str}), 1184 \mathrm{~cm}^{-1}: v_{\mathrm{C}-\mathrm{O}}(\mathrm{str}), 650 \mathrm{~cm}^{-1}$ :
$v_{\mathrm{C}-\mathrm{S}}(\mathrm{str})$.

The proton NMR spectrum of the product was recorded in chloroform using a $500 \mathrm{MHz}{ }^{1} \mathrm{H}$ NMR spectrophotometer. The results are given below and spectrum is shown in Figure 2. ${ }^{1} \mathrm{H}$ NMR: $10.32 \mathrm{ppm}(1 \mathrm{H}$, s: $-\mathrm{COOH}), 7.42 \mathrm{ppm}(2 \mathrm{H}, \mathrm{d}$ : aromatic proton a), $6.74 \mathrm{ppm}$ $(2 \mathrm{H}, \mathrm{d}$ : aromatic proton $\mathrm{b}), 4.81 \mathrm{ppm}\left(1 \mathrm{H}, \mathrm{s}: \mathrm{H}_{\mathrm{c}}\right), 3.65$ ppm $\left(2 \mathrm{H}, \mathrm{s}: \mathrm{H}_{\mathrm{d}}\right), 3.09$ ppm $\left(6 \mathrm{H}, \mathrm{s}: \mathrm{N}\left(\mathrm{CH}_{3}\right)_{2}\right.$ (Scheme 1).

\subsection{Synthesis and Characterisation of Lignin Functionalised with 2-(5-(4-Dimethylamino- benzylidin)-4-oxo-2-thioxo-thiazolidin-3-1)- acetic Acid}

The end hydroxyl functionalities of lignin were esterified with the free carboxyl group of 2-(5-(4-dimethylaminobenzylidin)-4-oxo-2-thioxo-thiazolidin-3-yl)acetic acid through DCC coupling using DMAP as the catalyst (Scheme 2).

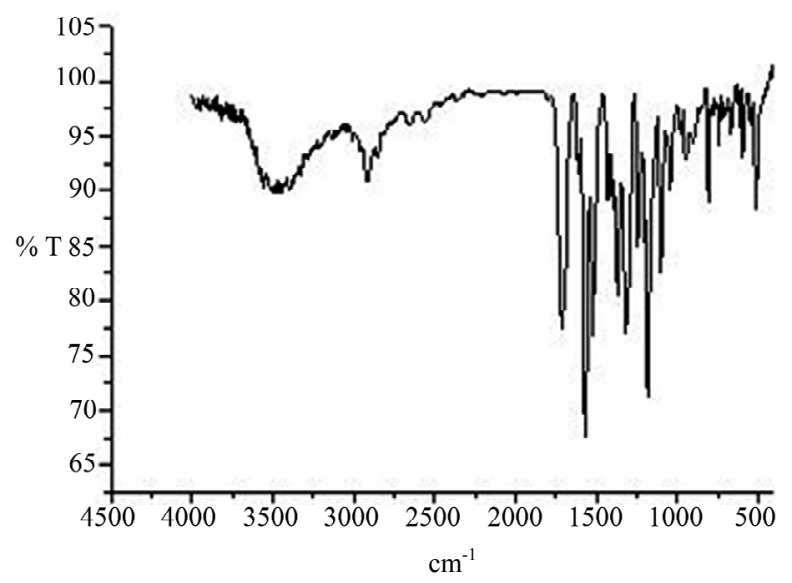

Figure 1. IR spectrum of 2-(5-(4-dimethy lamino-benzylidin)-4-oxo-2-thioxo-thiazolid-in-3-yl)-acetic acid.

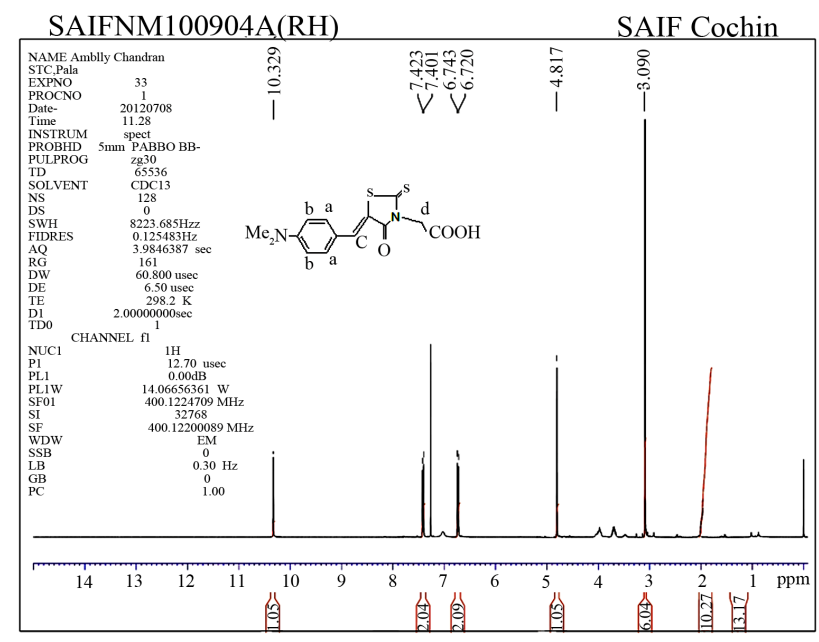

Figure 2. H1 NMR spectrum of 2-(5-(4-dimethylaminobenzylidin)-4-oxo-2-thioxo-thiazolidin-3-yl) acetic acid. 
IR spectrum was recorded in the solid state as $\mathrm{KBr}$ discs in the operating frequency range 4000-400 $\mathrm{cm}^{-1}$. IR(KBr): $3743 \mathrm{~cm}^{-1} v_{\mathrm{O}-\mathrm{H}}(\mathrm{str}), 2927 \mathrm{~cm}^{-1} v_{\mathrm{C}-\mathrm{H}}$ of $\mathrm{CH}_{2}$ aromatic, $1747 \mathrm{~cm}^{-1}: v_{\mathrm{C}=\mathrm{O}(\mathrm{str})}, 1535 \mathrm{~cm}^{-1}: v_{\mathrm{C}=\mathrm{C}}(\mathrm{str}), 1315 \mathrm{~cm}^{-1}$ : $v_{\mathrm{C}-\mathrm{N}}(\mathrm{str}), 1186 \mathrm{~cm}^{-1}: v_{\mathrm{C}-\mathrm{O}}(\mathrm{str}), 665 \mathrm{~cm}^{-1}: v_{\mathrm{C}-\mathrm{S}}(\mathrm{str})$. The IR spectrum is shown in Figure 3.

The proton NMR spectrum of the product was recorded in chloroform using a $500 \mathrm{MHz}{ }^{1} \mathrm{H}$ NMR spectrophotometer. ${ }^{1} \mathrm{H}$ NMR: $7.42-7.40 \mathrm{ppm}(2 \mathrm{H}, \mathrm{d}: \mathrm{Ha})$, 7.72 - 7.75 (2H, d: $\mathrm{Hb}), 6.70$ - 6.74 ppm (m: aromatic protons in lignin), $5.01 \mathrm{ppm}(1 \mathrm{H}$, s: unreacted phenolic $\mathrm{OH}$ in lig nin), 3.09 ppm $\left(6 \mathrm{H}, \mathrm{s}: \mathrm{N}\left(\mathrm{CH}_{3}\right)_{2}\right), 2.99$ ppm (s: alcoholic $\mathrm{OH}$ in lignin) $2.80 \mathrm{ppm}\left(2 \mathrm{H}, \mathrm{s}: \mathrm{H}_{\mathrm{d}}\right) .2 .22 \mathrm{ppm}$ (H, s: aliphatic proton c) [13-16]. The lignin aliphatic protons give multiplet at $1.6-1.9 \mathrm{ppm}$. The absence of a peak at 8-10 shows that the carboxylic proton is absent in the product and this confirms the coupling of free carboxyl group of the dye with hydroxyl functions of lignin. The spectrum is shown in Figure $4[13,14]$.

\subsection{Photoresponsive Studies}

The UV-visible spectrum was recorded in chloroform.

The $\lambda_{\max }$ of the dye was obtained at $474 \mathrm{~nm}$ (Figure 5(a) and the signal was shifted to $498 \mathrm{~nm}$ (Figure 5(c)) on attaching to lignin. The $\lambda_{\max }$ of lignin core was at 416

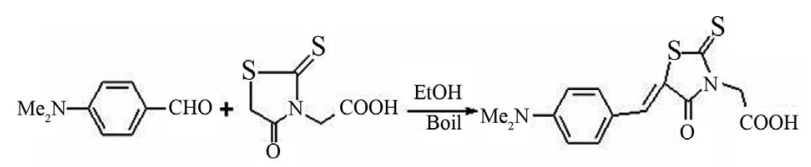

Scheme 1. Synthesis of 2-(5-(4-dimethylamino-benzylidin)4-oxo-2-thioxo-thiazolidin-3-yl)acetic acid。

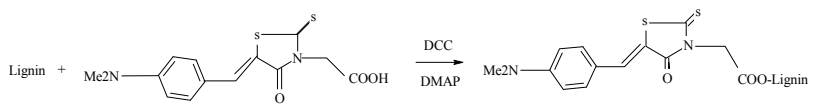

Scheme 2. Functional modification of lignin with 2-(5-(4-dimethylamino-benzylidin)-4-oxo-2-thioxo-thiazolidin-3-yl)acetic acid.

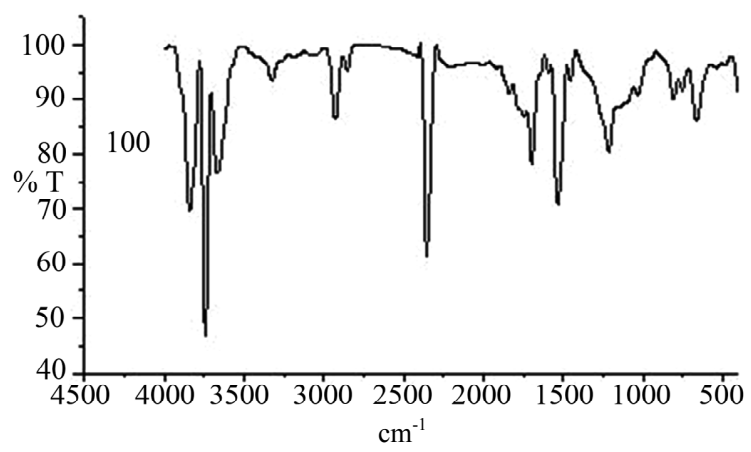

Figure 3. IR spectrum of lignin modified with 2-(5-(4-dimethylamino-benzylidin)-4-oxothioxo-thiazolidin-3-yl)- acetic acid.

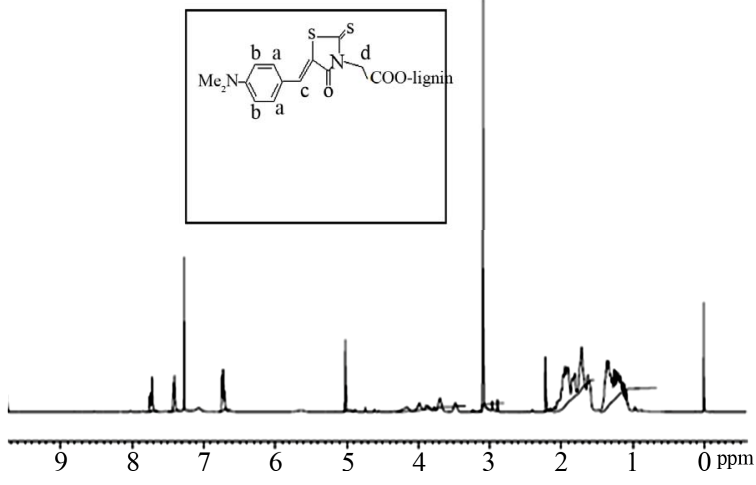

Figure 4. $\mathrm{H}^{1}$ NMR spectrum of lignin modified with 2(5-(4-dimethylamino-benzylidin)-4-oxo-2-thioxo-thiazolidin3-yl)-acetic acid.

nm (Figure 5(b)). The spectra were recorded using solutions of same molar concentrations and a strong increase in intensity was observed for the modified lignin system. Thus the photoabsorption properties of the chromophoric system becomes more efficient when attached to lignin and this was evident from the remarkable red shift and increase in intensity noted on attaching the dye to the core (Figure 5)

The light fastening properties of the chromophoric system and the lignin core functionalised with 2-(5(4-dimethylamino-benzylidin)-4-oxo-2-thioxo-thiazolidi n-3-yl)-acetic acid were investigated by irradiating these systems to visible radiant energy. The experiments were carried out in chloroform using same molar concentrations. In this experiment, the changes in the UV-visible absorption spectra as a function of time of irradiation were noted. The chromophoric system shows decrease in intensity as the time increases. The intensity of absorption at zero time was noted as 2.75 . After $1 \mathrm{hr}$ of irradiation the intensity was decreased to 2.5 and after five hours of irradiation, the intensity of absorption was notably decreased and it was 1.8. But in the modified lignin, there was no appreciable change in the intensity of absorption on irradiation for several hours. The intensity remained intact at 3.1. This shows that the stability of the chromophoric system greatly enhanced on attaching to lignin. The irradiation results are shown in Figure 6.

The fluorescence emission of lignin coupled with 2[5-(4-dimethylamino-benzylidine-4-oxo-2-thioxo-thiozol idine-3-yl]acetic acid was recorded in chloroform. The excitation wavelength was $450 \mathrm{~nm}$. The emission maximum for the coupled product was observed at $563 \mathrm{~nm}$, where as the emission maximum of pure chromo phoric system is at $554 \mathrm{~nm}$. The enhanced fluorescence emission of chromophore functionalised lignin core may be due to their ability to form intra molecular excimers. The fluorescence spectra of the pure chromophore and coupled lignin are shown in Figure 7. 


\section{Conclusion}

The paper reports the development of a novel nature friendly photoactive macro molecular system with electron modulation based on lignin. Lignin was functionally

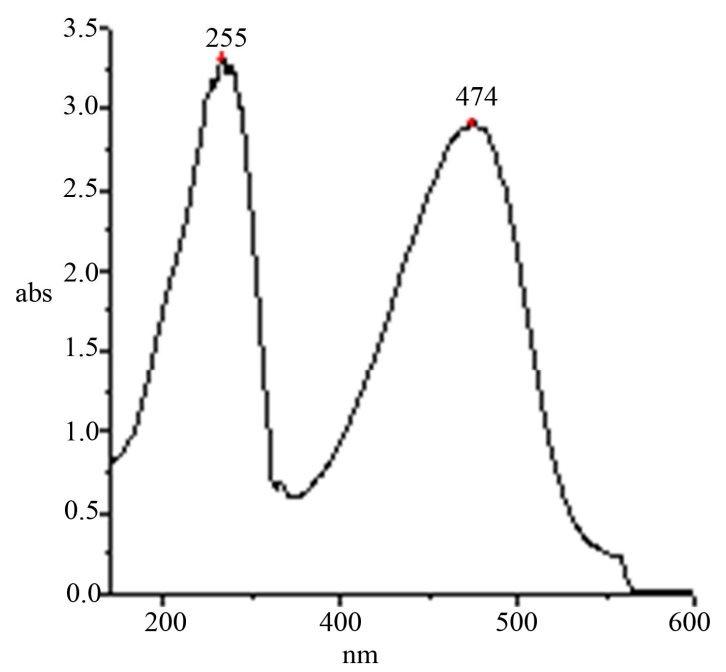

(a)

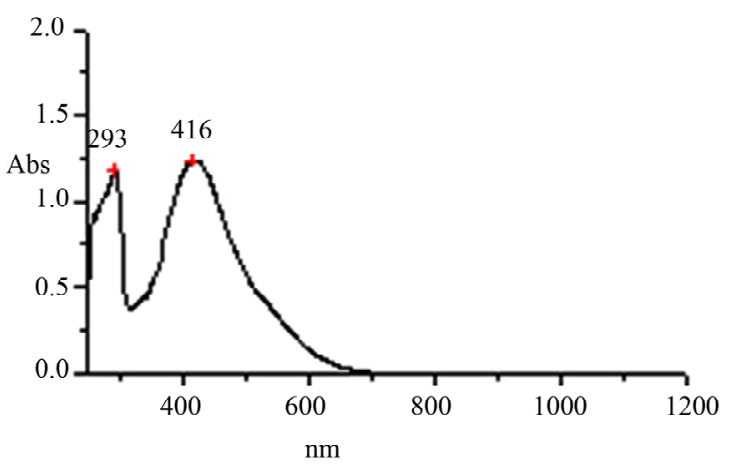

(b)

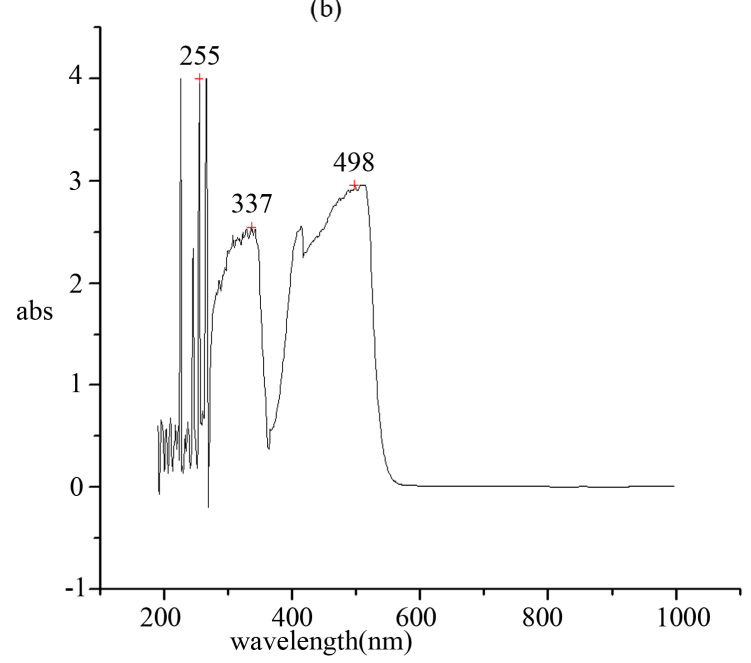

Figure 5. UV-visible spectrum of (a) 2-(5-(4-dimethy-lamino-benzylidin)-4-oxo-2-thioxo-thialidin-3-yl)acetic acid and (b) pure lignin and lignin modified with 2-(5-(4-dime-thylamino-benzylidin)-4-oxo-2-thioxo-thiazolidin-3-yl)acetic acid.

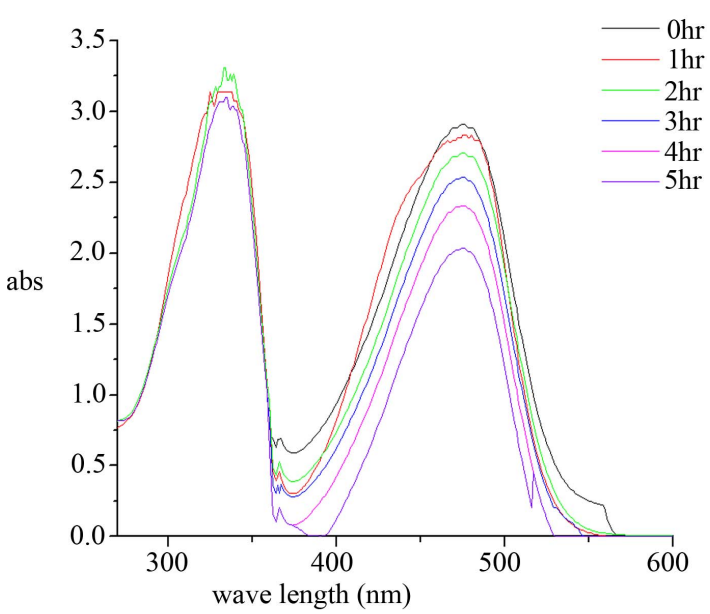

(a)

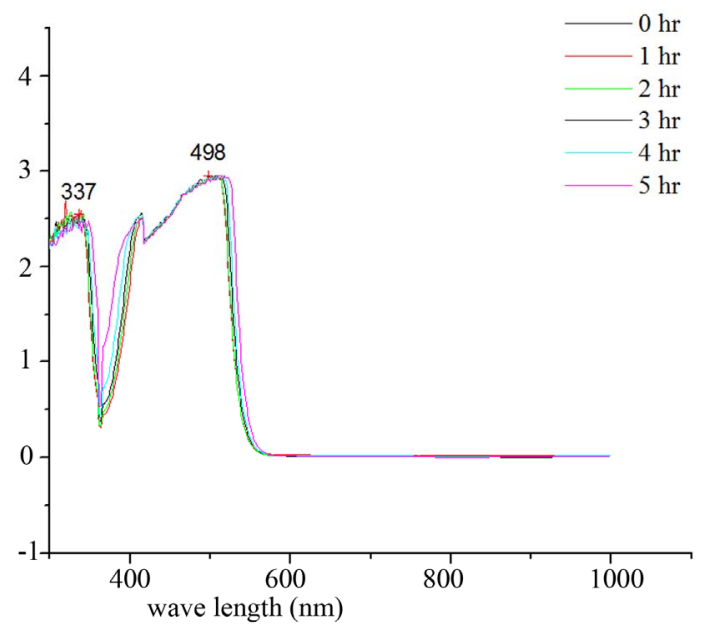

(b)

Figure 6. Light fastening studies of (a) 2-(5-(4-dimethylamino-benzylidin)-4-oxo-2-thioxo-thiazolidin-3-yl)acetic acid and (b) lignin modified with 2-(5-(4-dimethy lamino-benzylidin)-4-oxo-2-thioxo-thiazolidin-3-yl)acetic acid.

modified with a chromophoric system with push-pull electron modulation namely 2-(5-(4-dimethylamino-benzylidin)-4-oxo-2-thioxo-thiazolidin-3-yl)acetic acid by DCC coupling between the free carboxyl function of the chromophoric system on to the end hydroxyl functionalities of lignin. The products were characterised by spectroscopic methods. The photochemical properties of the coupled lignin were compared with the pure chromophoric system. The modified system shows a considerable red shift and remarkable increase in intensity of absorption and emission. On prolonged irradiation with visible radiant energy, the lignin functionalised with 2-(5-(4-dimethylamino-be-zylidin)-4-oxo-2-thioxo-thiaz olidin-3-yl) acetic acid showed excellent light stabilisa-tion properties compared to the monomeric dye. The strong red shift observed on binding the chromophoric system to lignin and the remarkable stability on irradiation provides a no- 


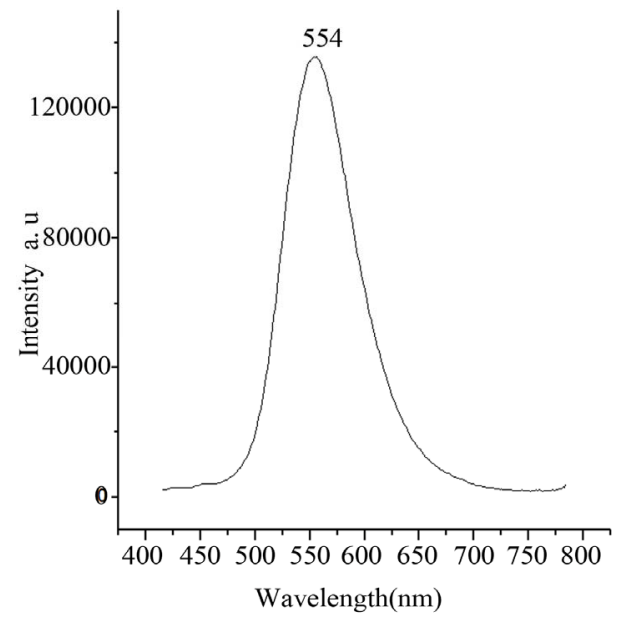

(a)

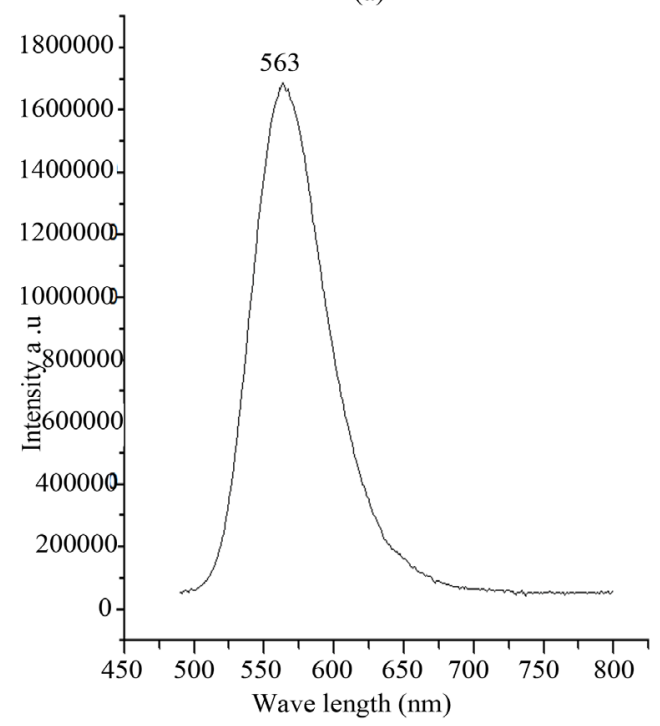

(b)

Figure 7. Fluorescence spectra of (a) 2-(5-(4-dimethylamino -benzylidin)-4-oxo-2-thioxo-thiazolidin-3-yl)acetic acid and (b) lignin modified with 2-(5-(4-dimethylamino-benzylidin) -4-oxo-2-thioxo-thiazolidin-3-yl)acetic acid.

vel photoresponsive system with excellent light fastening properties. Moreover it shows excellent emission behaviour which was demonstrated by recording the fluorescence spectra. Being a naturally occurring, inexpensive, water-soluble and environment friendly photoactive macromolecular system, the fuctionally modified lignin finds many applications in medicine, medical diagnosis, in paint and dye industry.

\section{Acknowledgements}

The authors thank the Department of Science and Technology (Ministry of Science and Technology), Govt. of India, New Delhi for financial support by awarding major research project No. SR/S1/OC-24/2006, dtd.26.10.2006.

\section{REFERENCES}

[1] J. F. Matte and J. Doucet, "Recent Developments in Lignin Utilization as Wood Adhesive: A Review," Cellulose Chemistry and Technology, Vol. 22, No. 1, 1988, pp. 7185.

[2] D. W. Sundstrom and H. E. Klei, "Uses of By-Product Lignins from Alcohol Fuel Processes," Biotechnology and Bioengineering Symposium, Vol. 12, 1982, pp. 45-56.

[3] J. Ralph, R. D. Hatfield, S. Quideau, R. F. Helm, J. H. Grabber and H.-J. G. Jung, "Pathway of P-Coumaric Acid Incorporation into Maize Lignin as Revealed by NMR," Journal of the American Chemical Society, Vol. 116, No. 21, 1994, pp. 9448-9456. doi:10.1021/ja00100a006

[4] J. Ralph, R. F. Helm, S. Quideau and R. D. Hatfield, "Lignin-Feruloyl Ester Cross-Links in Grasses. Part 1. Incorporation of Feruloyl Esters into Coniferyl Alcohol Dehydrogenation Polymers," Journal of the Chemical Society, Perkin Transactions, Vol. 1, 1995, pp. 29612969.

[5] J. Ralph and F. Lu, "The DFRC Method for Lignin Analysis. Part 6. A Modified Method to Determine Acetate Regiochemistry on Native and Isolated Lignins," Journal of Agricultural and Food Chemistry, Vol. 46, No. 11, 1998, pp. 4616-4619. doi:10.1021/jf980680d

[6] J. H. Grabber, J. Ralph and R. D. Hatfield, "Ferulate Cross-Links Limit the Enzymatic Degradation of Synthetically Lignified Primary Walls of Maize Digestibility," American society of Agronomy Inc, Maidson, 1993.

[7] S. Sarkanen, P. C. Teller, C. R. Stevens and J. L. Mccarthy, "Lignin. 20. Associative Interactions between Kraft Lignin Components," Macromolecules, Vol. 17, No. 12, 1984, pp. 2588-2597. doi:10.1021/ma00142a022

[8] K. V. Sarkanen and C. H. Ludwig, "Definition and Nomen Clature," In: Lignins: Occurance, Formation, Structure and Reactions, Wiley-Interscience, NewYork, 1971, pp. 1-16.

[9] W. Boerjan, J. Ralph and M. Baucher, "Lignin Biosynthesis," Annual Review of Plant Biology, Vol. 54, No. 1, 2003, pp. 519-546. doi:10.1146/annurev.arplant.54.031902.134938

[10] J. Ralph, K. Lundquist, G. Brunow, F. Lu, H. Kim, P. F. Schatz, J. M. Marita, R. D. Hatfield, S. A. Ralph, J. H. Christensen and W. Boerjan, "Lignins: Natural Polymers from Oxidative Coupling of 4-hydroxy Phenyl Propanoids," Phytochemistry Reviews, Vol. 3, No. 1, 2004, pp. 29-60. doi:10.1023/B:PHYT.0000047809.65444.a4

[11] D. C. C. Smith, "P-Hydroxy Benzoate Groups in the Lignin of Aspen (Populus Tremula)," Journal of the Chemical Society, 1995, pp. 2347-2351.

[12] D. C. C. Smith, "Ester Groups in Lignin," Nature, Vol. 176, No. 4475, 1955, pp. 267-268. doi:10.1038/176267a0

[13] J. R. Dyer, "Applications of Absorption Spectroscopy of Organic Compounds,” Phi Learning, New Delhi, 2009.

[14] W. Kemp, “Organic Spectroscopy," Macmillan, London, 1975. 